\title{
Strafloser Schwangerschaftsabbruch
}

\author{
Welchen rechtlichen Rahmen haben Spitäler beim Schwangerschaftsabbruch? \\ Das Schweizer Recht unterscheidet zwei Arten eines straflosen Abbruchs: zum einen \\ die Fristenregelung in den ersten 12 Wochen der Schwangerschaft und zum anderen \\ die Indikationsregelung.
}

Christian Peter

\section{«Eine schwere seelische Notlage droht, wenn die Frau leicht in einen dauerhaften psychischen Ausnahme- \\ zustand geraten könnte»}

\begin{abstract}
In einem kürzlich erschienenen Artikel in der Schweizerischen Ärztezeitung [1] haben Anne-Marie Rey und André Seidenberg festgehalten, dass sich öffentliche und private Spitäler bei den Schwangerschaftsabbrüchen nach der 12. Schwangerschaftswoche grosse $\mathrm{Zu}$ rückhaltung auferlegen und der weite Ermessensspielraum nicht ausgeschöpft wird.

Welchen rechtlichen Rahmen hätten die Spitäler überhaupt beim Schwangerschaftsabbruch? Dieser Frage will dieser Beitrag [2] nachgehen.

Das Schweizer Recht unterscheidet zwei Arten eines straflosen Schwangerschaftsabbruchs: Die sogenannte Fristenregelung, die einen relativ hindernisfreien Schwangerschaftsabbruch in den ersten 12 Wochen ermöglicht und die sogenannte Indikationsregelung, die dem ärztlichen Urteil eine sehr grosse Bedeutung beimisst.
\end{abstract}

Korrespondenz:

Dr. iur. Christian Peter Inselspital

Universitätsspital Bern Rechtsdienst

CH-3010 Bern

christian.peter@insel.ch

\section{Fristenregelung (Art. 119 Abs. 2 StGB)}

Damit ein Schwangerschaftsabbruch straflos bleibt, müssen folgende Voraussetzungen erfüllt sein:

- Der Eingriff muss innerhalb von 12 Wochen seit Beginn der letzten Periode (= 10 Wochen seit der Befruchtung) erfolgen. Die Dauer ist durch die Ärztin oder den Arzt nach dem Stand des aktuellen Wissens zu ermitteln [3].

- Der Eingriff muss durch einen zur Berufsausübung zugelassenen Arzt durchgeführt werden. Es muss sich also nicht zwingend um eine Chirurgin oder einen Gynäkologen handeln, aber er oder sie muss zur selbständigen Berufsausübung ermächtigt sein.

- Es muss ein schriftliches Begehren der schwangeren Frau (mit Unterschrift) vorliegen. Die Initiative muss von der Schwangeren selber ausgehen. Ist die Schwangere unmündig (jünger als 18 Jahre), aber urteilsfähig, so reicht ihr Verlangen aus [4].

- Die Schwangere muss eine Notlage geltend machen. Diese Notlage muss nicht an objektiven
Kriterien gemessen werden; es genügt, dass die Schwangere die Situation als Notlage empfindet und diese gegenüber der Ärztin oder dem Arzt geltend macht.

- Die Ärztin oder der Arzt muss mit der Schwangeren ein eingehendes Gespräch führen und sie beraten (Beratungsgespräch). Art. 120 Abs. 1 StGB konkretisiert den Inhalt dieses Gesprächs [5]. Eine Wartefrist zwischen Gespräch und Abbruch gibt es nicht.

Somit ist der Schwangerschaftsabbruch während der ersten 12 Wochen ohne grosse Auflagen zulässig und straflos.

\section{Indikationsregelung (Art. 119 Abs. 1 StGB)}

Nach Ablauf von 12 Wochen seit Beginn der letzten Periode ist ein Schwangerschaftsabbruch nur nach Vorliegen einer medizinischen oder sozialmedizinischen Indikation straflos.

\section{Voraussetzungen}

- Die Zustimmung der Schwangeren ist notwendig. Diese kann ausdrücklich, konkludent [6] oder mutmasslich erfolgen (analog der Einwilligung bei anderen medizinischen Eingriffen). Bei Urteilsunfähigkeit muss die Einwilligung durch den gesetzlichen Vertreter oder die gesetzliche Vertreterin erfolgen. Es ist zu beachten, dass Minderjährigkeit nicht zwingend mit einer Urteilsunfähigkeit einhergeht. Eine minderjährige Schwangere kann durchaus urteilsfähig sein, und die Zustimmung des gesetzlichen Vertreters oder der gesetzlichen Vertreterin ist nicht nötig [7].

- Der Eingriff muss durch einen zur Berufsausübung zugelassenen Arzt bzw. Ärztin durchgeführt werden. Es muss also nicht zwingend eine Chirurgin oder ein Gynäkologe sein, aber er oder sie muss zur selbständigen Berufsausübung ermächtigt sein. Der die Indikation stellende Arzt muss den Abbruch nicht selbst vornehmen, dies ist aber möglich.

- Es muss eine medizinische oder sozial-medizinische Indikation vorliegen. Diese ist - im Gegensatz zur Fristenregelung - nach ärztlichem Urteil zu bewerten. Der Arzt oder die Ärztin hat sich hier- 


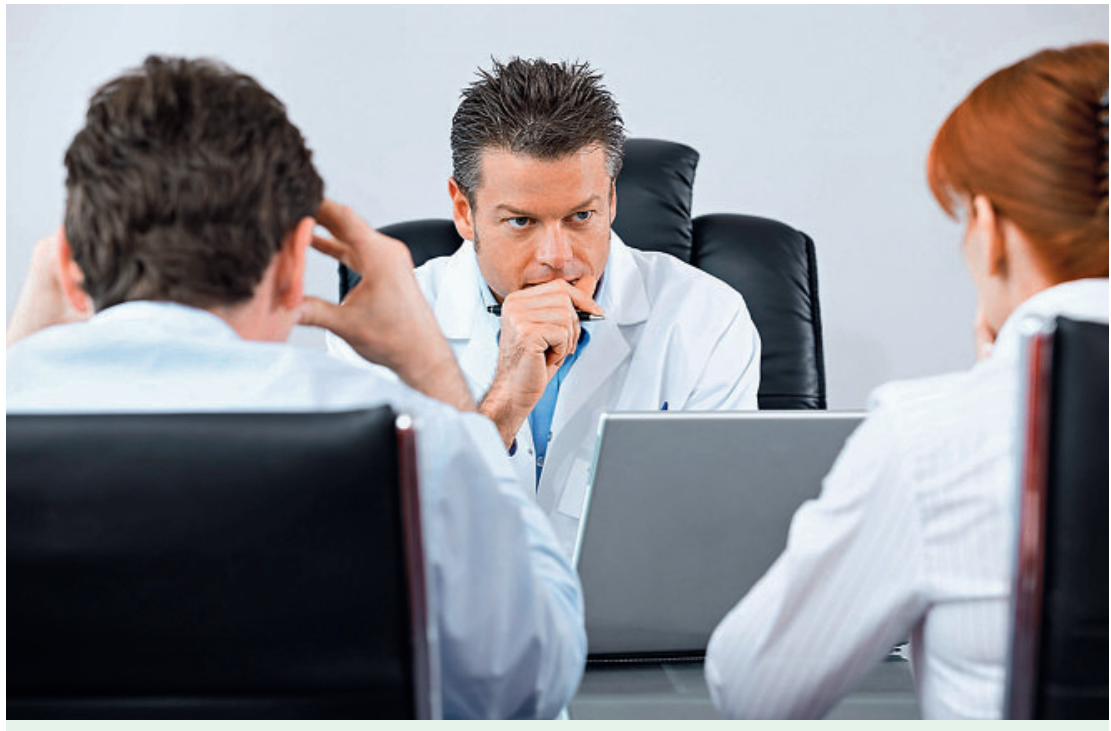

Bei der Indikationsregelung kommt dem ärztlichen Urteil zur Gefahr einer schweren körperlichen Schädigung oder dem Vorliegen einer seelischen Notlage grosse Bedeutung zu.

bei am neusten Stand der Wissenschaft und Praxis zu orientieren, wobei ihm oder ihr ein kaum überprüfbares ärztliches Ermessen zusteht. Die Gefahr muss umso grösser sein, je fortgeschrittener die Schwangerschaft ist.

- Indiziert ist der Schwangerschaftsabbruch, wenn er geeignet oder notwendig ist, um eine drohende, schwerwiegende körperliche Schädigung (inkl. drohende Lebensgefahr) abzuwenden. Neben der Suizidgefahr kommen Gesundheitsgefahren in Betracht wie beispielsweise das Auslösen oder Verstärken von Krankheiten. Keine Gefahr einer schwerwiegenden Schädigung kann in Komplikationen erblickt werden, die regelmässig mit einer Schwangerschaft einhergehen.

- Eine schwere seelische Notlage droht, wenn die Frau leicht in einen dauerhaften psychischen Ausnahmezustand geraten könnte. Der drohende psychische Zustand muss nicht einem Krankheitsbild entsprechen. Neben der Persönlichkeitsstruktur sind auch die gegenwärtigen und zukünftigen Lebensumstände der Schwangeren zu berücksichtigen. Es handelt sich somit um eine sozial-medizinische Gesamtindikation, die embryopathische, kriminologische, psychiatrische oder auch andere Gründe berücksichtigt. Würde die Pflege und Erziehung des kranken Kindes eine unzumutbare Überforderung der Mutter und der Familie bedeuten, läge eine embryopathische Indikation vor. In diese Gruppe würden auch die nicht überlebensfähigen Föten fallen, bei denen nach der Geburt eine Früheuthanasie (Sterbehilfe) gerechtfertigt wäre. War die Schwangere Opfer z. B. einer Vergewaltigung oder Schändung, die mit grosser Wahrscheinlichkeit die Schwangerschaft bewirkt hat, und ist hieraus eine schwere Notlage entstanden, liegt eine kriminologische Indikation vor.

\section{Fazit}

Bei der Fristenregelung müssen formelle Kriterien erfüllt sein, die in Art. 119 Abs. 2 und Art. 120 StGB niedergeschrieben sind. Bei der Indikationsregelung hingegen kommt neben den formellen Kriterien vor allem dem ärztlichen Urteil bezüglich der Gefahr einer schweren körperlichen Schädigung oder seelischen Notlage eine grosse Bedeutung zu. Ein Urteil, dem ein kaum überprüfbares Ermessen innewohnt.

\section{Literatur}

1 Rey A-M, Seidenberg A. Schwangerschaftsabbruch: die Praxis der Spitäler und Kliniken in der Schweiz. Schweiz Ärztezeitung. 2010;91(13/14):551-4.

2 Der Artikel beruht auf den Ausführungen im Basler Kommentar Strafrecht II - Schwarzenegger C. und Heimgartner S., Art. 119 f.

3 Diese Frist ist im internationalen Vergleich sehr kurz bemessen und führt im Resultat dazu, dass der Schwangeren nach Ausbleiben der Periode lediglich eine Überlegungsfrist von knapp 2 Monaten bleibt.

4 Es ist umstritten, ob die Schwangerschaft einer minderjährigen und urteilsunfähigen Frau, gestützt auf Abs. 2, abgebrochen werden kann. In solchen Fällen sollte zur Sicherheit ein Unterbruch nach der Indikationsregelung (siehe folgend) durchgeführt werden.

5 Verlangt werden ein eingehendes Gespräch, die Beratung über die gesundheitlichen Risiken des Eingriffs und Auskunft über die Möglichkeit, das geborene Kind zur Adoption freizugeben. Ferner muss der Schwangeren gegen Unterschrift ein Leitfaden ausgehändigt werden, der folgendes enthält: ein Verzeichnis der kostenlos zur Verfügung stehenden Beratungsstellen und ein Verzeichnis von Vereinen und Stellen, die moralische und materielle Hilfe anbieten. Bei Schwangeren unter 16 Jahren hat man sich zu vergewissern, dass sie sich an eine auf Jugendliche spezialisierte Beratungsstelle gewandt haben.

6 Als konkludent wird eine Willenserklärung bezeichnet, die ohne ausdrückliche Erklärung durch schlüssiges Verhalten abgegeben wird. Die Willenserklärung wird also aus den Handlungen des/der Erklärenden abgeleitet.

7 Vgl. Peter C. Die Einwilligung von Minderjährigen in medizinische Eingriffe. Schweiz Ärztezeitung. 2008;89(36):1539-40. 